\title{
Secure sensor networks for perimeter protection
}

\author{
Sasikanth Avancha *, Jeffrey Undercoffer, Anupam Joshi, John Pinkston \\ Department of Computer Science and Electrical Engineering, University of Maryland, Baltimore County, 1000 Hilltop Circle, \\ Baltimore, MD 21250, USA
}

\begin{abstract}
Sensor networks have been identified as being useful in a variety of domains to include the battlefield and perimeter defense. We motivate the security problems that sensor networks face by developing a scenario representative of a large application class where these networks would be used in the future. We identify threats to this application class and propose a new lightweight security model that operates in the base station mode of sensor communication, where the security model is mindful of the resource constraints of sensor networks. Our application class requires mitigation against traffic analysis, hence we do not use any routing mechanisms, relying solely on broadcasts of end-to-end encrypted packets. Our model extends the broadcast range of the base station model by utilizing nodes adjacent to the base station as an intermediary hop. Additionally, our model detects and corrects some classes of aberrant node behavior. We have simulated our model and present simulation results.
\end{abstract}

(c) 2003 Elsevier B.V. All rights reserved.

Keywords: Wireless sensor network; Energy-efficient security; Authentication; Confidentiality; Perimeter protection

\section{Introduction}

Improvements in wireless networking and micro-electro-mechanical systems (MEMS) are contributing to the formation of a new computing domain-distributed sensor networks. These adhoc networks of small, fully programmable sensors will be used in a variety of applications: on the battlefield, as medical devices, in equipment maintenance and in perimeter security systems $[1,2]$. These distributed sensor networks are character-

\footnotetext{
${ }^{*}$ Corresponding author. Tel.: +1-410-455-2668; fax: +1-410455-3969.

E-mail addresses: savanc1@cs.umbc.edu (S. Avancha), junder2@cs.umbc.edu (J. Undercoffer), joshi@cs.umbc.edu (A. Joshi), pinkston@cs.umbc.edu (J. Pinkston).
}

ized by limited power supplies, low bandwidth, small memory sizes and a different traffic model. Whereas the traffic model of mobile ad-hoc networks is typically many-to-many, the traffic model of a sensor network is more of a hierarchical model and/or many to one. Generally, MEMS are significantly more resource constrained than typical "mobile" or "handheld" devices. A node in a sensor network may or may not have computing requirements. In the case where computations are required, if the cost of a communication is less than the cost of the computation, the computation may be replaced by a request to a computationally robust central location. The threat to a sensor network is different from the threat to a mobile adhoc network. As such, existing network security mechanisms, including those developed for Mobile Ad-Hoc Networks, are a poor fit for this domain. 
Research into authentication and confidentiality mechanisms designed specifically for sensor data and network control protocols is needed. Given the fact that little prior work ([3] being the exception) exists in this space, there is a need both to identify the problems and challenges and propose solution techniques.

In this paper, we motivate the security problems that sensor networks face by developing a scenario representative of a large application class where such networks would be used in the (near) future. The specific application that we consider in this work is perimeter protection for high government officials. We identify the threats and vulnerabilities to this application, starting from the radio layer and progressing to the application layer. This paper details why security mechanisms that are presently used in mobile ad-hoc environments are inadequate or not appropriate for sensor networks designed for this application. We then describe a new security model that serves as a countermeasure to the identified threats. Our model of sensor network utilizes the central, fixed base station paradigm and is mindful of the resource constraints of sensor networks. We have implemented our model in SensorSim [4] and present our simulation results.

The remainder of this paper is organized as follows. Section 2 details related research in the area of security for sensor networks. Section 3 details our class of application, stating how sensor networks are a solution. Section 4 details our security protocol for sensor networks designed for this application class. Section 5 details our implementation and simulation results. We state our plans for future work in Section 6.

\section{Related work}

There is relatively little work in the area of securing sensor networks. Like their mobile ad-hoc counterparts, sensor networks lack a fixed infrastructure and the topology is dynamically deployed. Addressing the security of mobile ad-hoc networks, Yi et al. [5] point out that if the routing protocol can be subverted and messages altered in transit, then no amount of security on the data packets can mitigate a security threat at the application layer. Consequently, they introduce "Security Aware Ad-hoc Routing" (SAR). SAR characterizes and explicitly represents the trust values and relationships associated with ad-hoc nodes and uses these values to make secure routing decisions. They address two problems: Ensuring that data is routed through a secure route composed of trusted nodes and the security of the information in the routing protocol. To motivate their scenario, they use the example of two military generals wishing to communicate via an ad-hoc network using a generic form of the $\mathrm{Ad}-\mathrm{Hoc} \mathrm{On}$ Demand Distance Vector Routing (AODV) protocol. They employ a route discovery protocol where only nodes with a security metric equivalent to the sender and receiver participate in the routing process. Their work appears to be based on the Bell-La Padula Confidentiality Model [6]. Their model, however, is dependent on self-enforcement where nodes with a lower than required security level voluntarily opt out of participating in the hop-by-hop routing process.

Perrig et al. [3] introduce "SPINS: Security Protocols for Sensor Networks" comprised of Sensor Network Encryption Protocol (SNEP) and $\mu$ TESLA. The function of SNEP is to provide confidentiality (privacy), two-party data authentication, integrity and freshness. $\mu$ TESLA is to provide authentication to data broadcasts. SPINS presents an architecture where the base station accesses nodes using source routing. We describe SPINS in greater detail in Section 4.5 and also compare it to our model.

\section{Perimeter protection as a class of sensor appli- cations}

We motivate our application class of perimeter security by considering the following scenario.

In today's geopolitical climate the threat posed to high-level government officials is overwhelming, particularly when they are engaged in official business and traveling outside of their home countries.

Consider the typical case of a country's foreign minister. She travels extensively, often on the spur 
of the moment, and sometimes to destinations that are hostile. Due to the nature of her office, her security detail may not have had adequate time to conduct a detailed advance and install the requisite security controls.

The foreign minister faces a number of threats. Physical threats include poisoning from radiation, chemical or biological toxins. These are in addition to threats from explosives and individuals utilizing small arms or other military ordnance. More insidious threats are posed by collection efforts aimed at both the substance of her agenda and at analyzing security controls in order to compromise them at some later time.

Such perimeter security applications represent a vast class of "monitoring and responding" type applications for which sensor networks will be used. We believe that a solution designed to mitigate the aforementioned threats will also cover the broad spectrum of all threat models that such applications face.

Our application, and its attendant security model, may be abstracted and applied to other scenarios where concentric circles of perimeter protection need to be temporarily established. Examples of other applications are placing alarm fields on a border and at ports of entry in order to prevent the smuggling of hazardous materials and munitions and to prevent illegal entry. For example, suppose information is developed leading to the suspicion that some person or persons unknown will be attempting to move fissionable material across a remote section of a country's border. Furthermore, suppose that this material produces a signature which is only discernible at distances of $20 \mathrm{~m}$ or less. Such a scenario is within the realm of possibility. Moreover, it is conceivable that those behind such an operation could be leaking spurious information so as to cause the authorities to take precautionary measures, only to study, analyze and circumvent future preventive measures. This is in effect a "dry run" in preparation for the actual event. A sensor network could be rapidly deployed in order to prevent and apprehend those involved in such an event. However, the underlying architecture of the sensor network must be able to withstand probes and analyses in order to remain effective over time.

\subsection{Sensor technology as a solution}

Sensors are still some time away from actual mass fabrication and use. Current smartsensor prototypes, such as the Berkeley Renee Mote, have a larger footprint and are generally restricted to the following sensor types: accelerometers, microphones, light, motion and magnetometers. We envision a design inclusive of these sensors types; however, our proposed application assumes a (not so) futuristic scenario that include sensors that test for nitrates (explosives), chemical toxins, biological toxins and radiation. For example, sensors testing for motion and sound would be placed on rooftops, which provide an assailant(s) with a vantage point. Sensors testing for radiation would be placed in areas frequented by the protectee, while sensors testing for chemical and biological toxins would be placed in airways that feed into areas frequented by the protectee. Our colleagues at UMBC in the Department of Chemical and Biochemical Engineering are deploying such sensors [7], and we feel that such sensors will eventually be integrated with sensor prototypes.

Our contribution in this area is the creation of lightweight techniques for securing existing sensor network routing and data movement approaches, such as directed diffusion [8], SPIN [9] and data dissemination $[10,11]$. We assume the computational capability and memory requirements typical of those provided by sensors designed for the applications described above. We further assume that the base station is fixed, always attended and is in a secure location. We note, that in this work we make no attempt to counter the threat from a widespread denial of service attack against the RF layer. As pointed out in [12], such attacks are fairly straightforward to mount against fixed frequency $\mathrm{RF}$ communication links that are found in the sensors. Defending against them requires changes to the RF layer of the sensor, such as the use of spread spectrum techniques, which can mitigate against an RF level DoS attack. In our scenario, and many others, a denial of service is in itself an alarm.

The typical security problems that we might expect in the above scenario include: 
(i) Passive Information Gathering: If communications between sensors, or between sensors and base stations, are in the clear, then an intruder with an appropriately powerful receiver and well designed antenna can easily pick off the data stream. If information has to be encrypted, then "how" is the open question. In other words, which cryptographic approaches will be best, given the resource constraints and the routing paradigm employed by the sensor?

(ii) Subversion of a Node: A particular sensor might be "captured", and information stored on it (such as the key) might be obtained by an adversary. If a node has been compromised, then how to exclude that node, and that node only, from the sensor network is at issue. A number of vendors of cryptographic modules, such as smart cards, that store keying material are compliant with FIPS-140-2 [12], which may be adapted to the security solutions for sensor networks.

(iii) False Node: An intruder might "add" a node to the system that feeds false data or prevents the passage of true data. While such problems with malicious hosts have been studied in distributed systems, as well as ad-hoc networking, the solutions proposed there (group key agreements, quorums and per hop authentication) are in general too computationally demanding to work for sensors.

(iv) Legitimate Addition of a Node to an Existing Sensor Network: If a node needed to be replaced or another node needed to be added to an existing sensor network, securely integrating the new node into the existing network is at issue.

\subsection{Assumptions}

We make the following assumptions when applying sensor technology as a solution to our application class of perimeter security:

- The base station is computationally robust, having the requisite processor speed, memory and power to support the cryptographic and routing requirements of the sensor network. As stated above, the base station is in a fixed, secure location and is always attended. Hence, it is considered to be within a trusted computing environment. The assumptions of secure location and constant attendance are not valid for the individual sensor nodes.

- Key management and re-keying mechanisms are not necessary because the base station, which holds the cryptographic keys of all sensor nodes, is secure.

- The communication paradigm is one-to-one, not one-to-many or many-to-one. Even though all messages are broadcast at the routing and MAC levels, each message has an intended recipient. The threat of traffic analysis is mitigated by the use of end-to-end encryption of all transmissions.

- The physical security protocol of our application class follows the traditional model of concentric circles of protection, where the inner circle is resource rich. Conversely, as the distance from the inner circle increases the controls and mechanism become more general and are deployed in fewer numbers.

\section{Security model}

Security is a broadly used term encompassing the characteristics of authentication, integrity, confidentiality, non-repudiation and anti-playback. In the case of our sensor network the security requirements are comprised of authentication, integrity, confidentiality, anti-playback and resilience against traffic analysis. The recipient of a message needs to be able to be unequivocally assured that the message came from its stated source. Similarly, the recipient needs to be assured that the message was not altered in transit and that it is not an earlier message being replayed in order to veil the current environment. Finally, all communications need to be kept secret so that eavesdroppers cannot intercept, study and analyze, and devise counter measures in order to circumvent the purposes of the sensor network.

Our approach to defining a security model for sensor networks is resource driven and factors in the trade offs between levels of security and the 
requisite power and computational resources. Primarily, we envision a scenario where a protected perimeter based on sensors is dynamically deployed. However, similar scenarios could be envisioned in an environment where the topology is well known in advance and the sensor network is pre-configured. Our operating paradigm is where data is reported to a computationally robust central location such as a base station or network controller.

\subsection{Single collection and authentication point (base station) paradigm}

Consider the family of sensor routing protocols where each sensor communicates either directly or indirectly with a base station. In turn, the base station correlates and aggregates information from each sensor. Accordingly, the base station will need to verify the authenticity of the sensor, the integrity of the communication and ascertain that it is not a replay of an earlier communication. Recall the assumption that the base station is computationally robust and secure. In our protocol each sensor $\boldsymbol{j}$ shares a unique 64-bit key $\boldsymbol{K e y}_{j}$ and a 64-bit common key $\boldsymbol{K e y}_{\mathrm{BS}}$, with the base station. Our protocol provides for a two-hop scenario where the range of a base station is extended, employing nodes that are adjacent to the base station to serve as intermediaries for non-adjacent nodes. Fig. 1 depicts an example of such a sensor network topology.

Our goal is to provide confidentiality and integrity to the data, to authenticate the sender, to prevent replay attacks and to prevent traffic analysis; consequently, the entire communication is encrypted end-to-end. All communications consist of a preamble, header and payload. The preamble is empty if the communication originates from the base station and is directed to a sensor, otherwise it contains the address of the sending node. The header contains the recipient's address, nonce and a command and is encrypted under key $\boldsymbol{K e y}_{j}$, which is shared between the base station and node $\boldsymbol{j}$. The payload contains data exchanged between the node and the base station. As will be explained, the payload is encrypted under the shared key of the destination node, which may be different from the

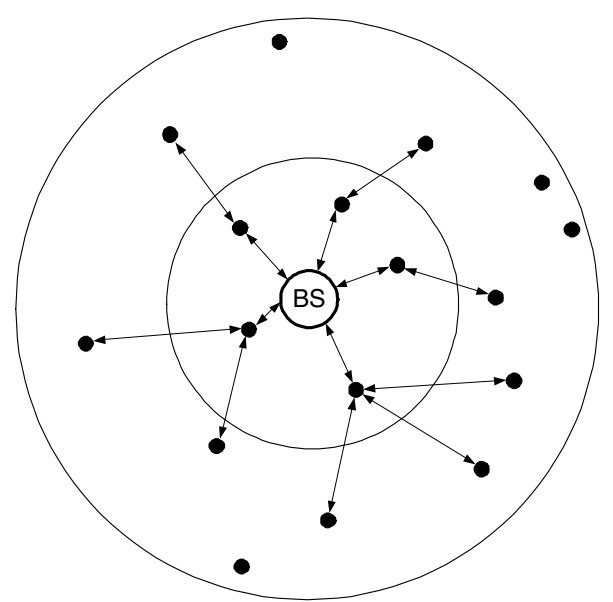

Fig. 1. Example network topology.

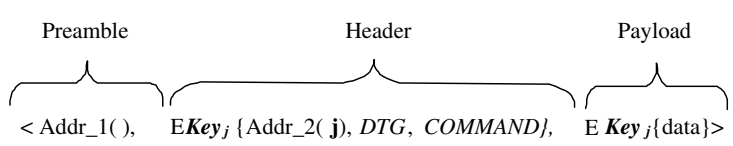

(a)

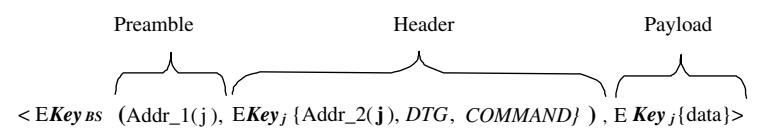

(b)

Fig. 2. (a) Base station to sensor message format; (b) sensor to base station message format.

key used to encrypt the header. This difference comes into play when the communication needs to be relayed by an intermediate node. Fig. 2 depicts the communication format.

Where

- Addr_1 is empty if the communication is from the base station to a sensor.

- Addr_1 contains the address of the transmitting node if the communication is directed to the base station. A non-empty Addr_1 field enables the base station to immediately select the correct key, instead of trying keys until it locates the correct one.

- Addr_2 contains the address of the destination node if the communication is from the base 
station to a node. If the communication is from a node to the base station Addr_2 will contain the address of the sending node.

- DTG is the date-time-group and is a nonce used to prevent replay attacks.

- COMMAND describes the contents of the message to the recipient.

Accordingly, a communication from the base station to a node $\boldsymbol{j}$ is of the form depicted in Fig. 2(a), and a communication from a node $\boldsymbol{j}$ to the base station is of the form: EKey $_{\mathrm{BS}}$ (Preamble, $\boldsymbol{E K e y}_{j}($ Header $\left.)\right), \boldsymbol{E K e y}_{j}($ Payload). On receipt, the base station decrypts the communication, uses the address contained in the preamble to select the key shared with the node and decrypts the message.

The use of double encryption prevents the disclosure of the number of nodes in the network and their addresses, consequently preventing traffic analysis. Should a single node be compromised and the its keys disclosed, the network will be able to function albeit the unique addresses of each node will be disclosed.

\subsection{Topology discovery and network setup}

The base station is deployed with the unique ID, symmetric encryption key of each node in the sensor network and a master key, $\boldsymbol{K e y}_{\mathrm{BS}}$. Similarly, each node is deployed with the unique key, $\boldsymbol{K} \boldsymbol{e} \boldsymbol{y}_{j}$ that it shares with the base station and $\boldsymbol{K e y}_{\mathrm{BS}}$. As in SPINS, its clock is synchronized with the base station's clock. We note that sensors do not obtain their keys "over the air" from the base station; rather every sensor is programmed with a unique key. Upon initialization of the sensor network the base station learns the network topology, creates and optimizes a routing table and provides a mechanism to non-adjacent (out of radio range) nodes that enables them to securely communicate with the base station.

At start up, the base station sends a HELLO message to each node $\boldsymbol{j}$. A node $\boldsymbol{j}$ attempts to decrypt the header of this message using its key. Successful decryption implies that the message was intended for $\boldsymbol{j}$. Node $\boldsymbol{j}$ responds to the HELLO message with a HELLO-REPLY message that it constructs using the message format in Fig. 2(b).
On receiving the encrypted HELLO-REPLY message, the base station decrypts the preamble using $\boldsymbol{K} \boldsymbol{e} \boldsymbol{y}_{\mathrm{BS}}$. It obtains $\boldsymbol{j}$ 's address from Addr_2, uses it to determine $\boldsymbol{K} \boldsymbol{e} \boldsymbol{y}_{j}$ from its Key Table and decrypts the header. If the header contains a valid DTG and the HELLO-REPLY command, then $\boldsymbol{j}$ is adjacent to the base station and the latter adds that node to its route table. Those nodes that did not reply are assumed to be two hops away and non-adjacent to the base station.

For these non-adjacent nodes $\boldsymbol{k}$, the base station sends a HELLO message via each adjacent node $\boldsymbol{j}$. In order to accomplish this, the base station places the header and payload of this message in the payload of a message containing the $R E$ $L A Y$ command and directs the RELAY message to a particular adjacent node $j$. In a $R E L A Y$ message, the header is encrypted under $\boldsymbol{K} \boldsymbol{e} \boldsymbol{y}_{j}$ and the payload, intended for $\boldsymbol{k}$, is encrypted under $\boldsymbol{K} \boldsymbol{e} \boldsymbol{y}_{k}$.

On receipt of the RELAY message, node $j$ prepends the original preamble to the payload and transmits the new message. The header of the message received by $\boldsymbol{k}$ contains a HELLO command and the payload contains a mechanism that will be used by $\boldsymbol{k}$ to reach the base station through the intermediate (adjacent) node $\boldsymbol{j}$.

This mechanism, referred to as $\Psi$, is a header containing the RELAY command encrypted under $\boldsymbol{K} \boldsymbol{e} \boldsymbol{y}_{j}$. To respond to the HELLO message, $\boldsymbol{k}$ constructs a HELLO-REPLY message encrypting it under $\boldsymbol{K} \boldsymbol{e} \boldsymbol{y}_{k}$ and places it in the payload. The preamble containing Addr_2( $k)$ and $\Psi$ are prepended to the payload and the message is transmitted. In turn, the adjacent node $\boldsymbol{j}$ receives the transmission, decrypts the header and upon seeing the RELAY command, prepends the preamble, appends $\operatorname{EKey}_{\mathrm{BS}}(j)$ to the payload and transmits it to the base station. Once the base station discovers which nodes are adjacent to it and all of the paths by which the non-adjacent nodes are reachable, it optimizes its route table so as to not overburden an adjacent node with the task of relaying messages. If the optimization process results in a different route, the base station sends the affected non-adjacent node an updated $\Psi$.

Fig. 3 shows the format of the HELLO and HELLO-REPLY message types exchanged between the base station and adjacent nodes. Fig. 4 
HELLO: Preamble; EKey $_{\mathrm{j}}\{$ Header $\}$; Payload Preamble: Addr_1()

Header: Addr2_(j), DTG, HELLO

Payload: $\varphi$

HELLO-REPLY: EKey ${ }_{\mathrm{BS}}\{$ Preamble, Header $\}$; Payload Preamble: Addr_2(j)

Header: EKey ${ }_{j}\{$ Addr_2(j), DTG, HELLO-REPLY\}

Payload: $\varphi$

Fig. 3. Messages exchanged between base station and adjacent nodes.

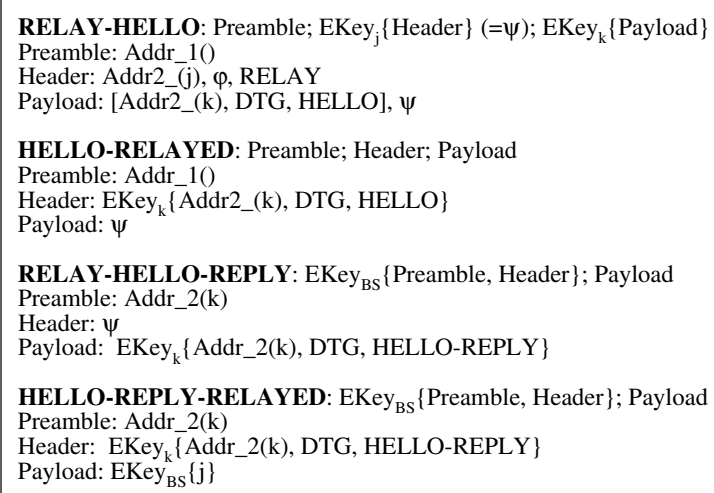

Fig. 4. Messages exchanged between base station and nonadjacent nodes.

shows the formats of these two message types exchanged between the base station and a nonadjacent node via an adjacent node. The secure topology discovery and network setup algorithm is presented in Fig. 5.

Three tables are maintained by the base station to help maintain and repair the network as required.

The Route Table contains the primary route, indicated by an *, and alternate routes to a node. An entry of the form A:( ) indicates that the node is directly connected to the base station whereas an entry such as D:(A) indicates that $A$ is an intermediate node between the base station and node D.

The Key Table contains the unique key shared by node $\boldsymbol{j}$ and the base station.

The Activity Table contains the most recent Date Time Group (DTG) received by the base station from a particular node, a count $(X)$ of

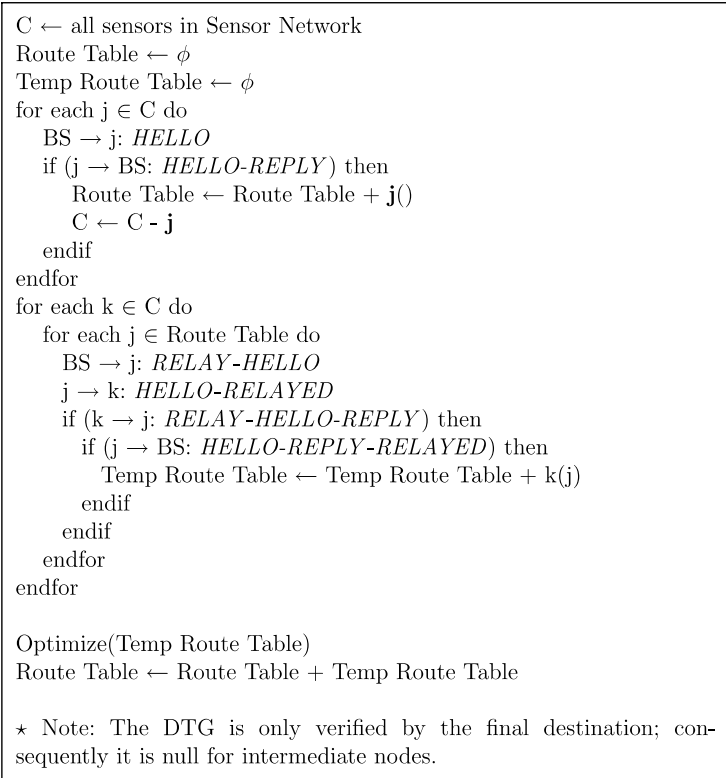

Fig. 5. Secure topology discovery and network setup protocol.

corrupted messages sent by the node, and a count $(Y)$ of other nodes dependent upon this node to relay messages. The values of $X$ and $Y$ are used to detect aberrant behavior on the part of an individual node.

These tables and other symbols used in the network setup and topology discovery and the network repair protocol are illustrated in Fig. 6.

We use DES [13] with a 64-bit key in Cipher Feedback (CFB) mode [14] (also known as cipher text auto-key) for data encryption. This type of cryptosystem is also detailed in $[15,16]$. We selected DES because it is a well-known and standardized encryption algorithm. It is resilient to cryptanalysis and its only known vulnerability is to a brute-force attack. However, to effectively implement a brute-force attack, some degree of knowledge about the expected plaintext is required in order to distinguish between plaintext and garble. Additionally, [17] has shown that DES can be optimized to reduce power consumption by $66 \%$. For the application class of perimeter protection, the encryption algorithm need only withstand a brute-force attack for the life of the sensor network, which we expect to be of the order of weeks. 


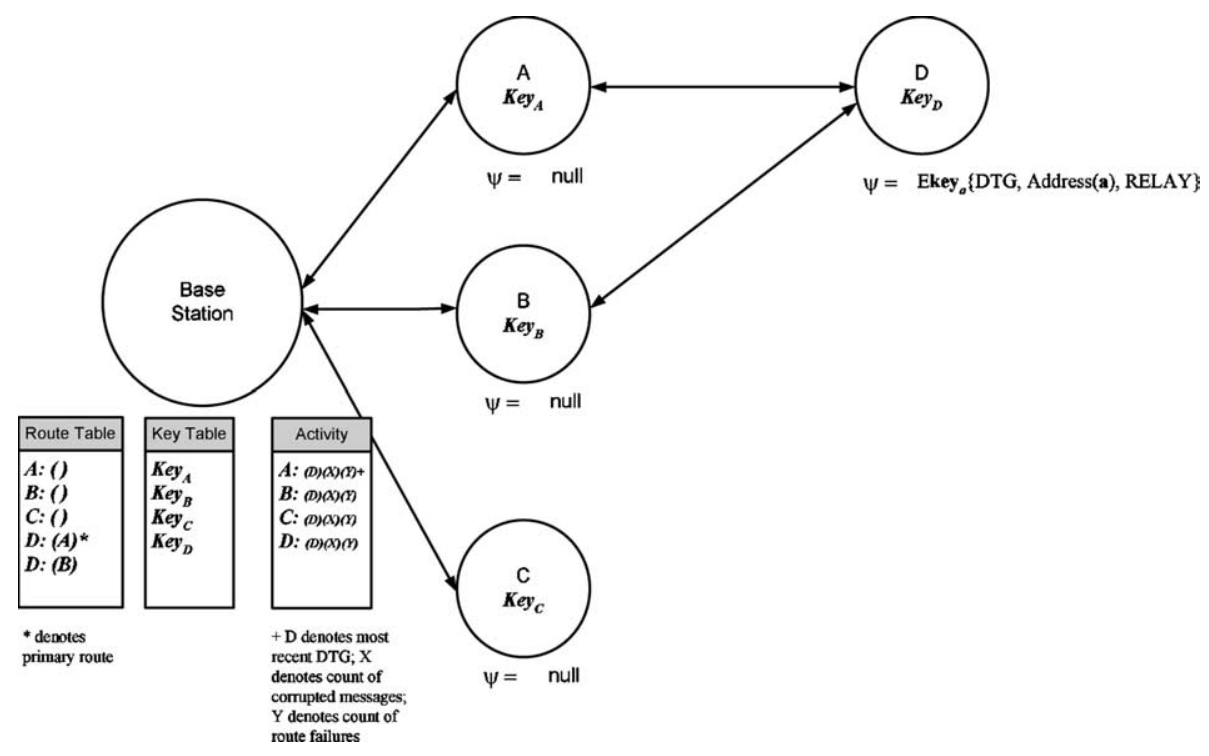

Fig. 6. Detailed example network topology.

To prevent traffic analysis, the entire communication is encrypted. Accordingly, a node will need to decrypt all communications that it "hears". This adds very little overhead because when the node decrypts the first 64 bits of the message, the recipient's address (Addr_2) is revealed. If a valid address is present then the node will continue to decrypt the message, otherwise it will discard it.

As previously stated, authentication is achieved through the use of a shared secret, which is the 64bit key $\boldsymbol{K} \boldsymbol{e} \boldsymbol{y}_{j}$, shared between the base station and node $\boldsymbol{j}$. Message integrity is achieved through the selection of an encryption algorithm that exhibits strong properties of diffusion and confusion. Accordingly, an attack aimed at altering the message will only be against the form of the message and not its substance. Anti-playback is achieved by the use the Date-Time-Group. Finally, privacy is achieved as a result of encrypting all communications.

\subsection{Inserting additional nodes into the network}

The insertion of an additional node into the existing sensor network is easily accomplished. In our model the unique identity and the key $\boldsymbol{K} \boldsymbol{e} \boldsymbol{y}_{m}$ of some node $\boldsymbol{m}$ to be added are loaded into the base station, the new node's clock is synchronized with that of the existing network and the base station repeats the topology discovery algorithm, explicitly looking for the new node. Once the new node is discovered, the routing table is re-optimized. For the perimeter protection application class, the need to insert additional nodes into the network is not expected to be high, given the limited duration for which protection is required.

\subsection{Isolating aberrant nodes}

An aberrant node is one that is not functioning as specified. Identifying and isolating aberrant nodes that are serving as intermediate nodes is important to the continued operation of the sensor network.

A node may cease to function as expected for several reasons, to include:

1. It has exhausted its source of power.

2. It was damaged.

3. It is dependent upon an intermediate node and is being blocked because the intermediate node has fallen victim to 1 and 2 above.

4. It is dependent upon an intermediate node and is being deliberately blocked because the intermediate node has been compromised. 
5. An intermediate node has been compromised and it is corrupting the communication by modifying data before forwarding it.

Our protocol effectively mitigates against the class of attack/failure where an intermediate node is involved. The protocol for the detection of aberrant nodes is presented in Fig. 7.

Periodically, the base station checks the activity table associated with a node, testing for a prolonged period of inactivity, $\Delta$. If the node is directly connected (i.e., it does not rely upon an intermediate node) this could be evidence of ab-

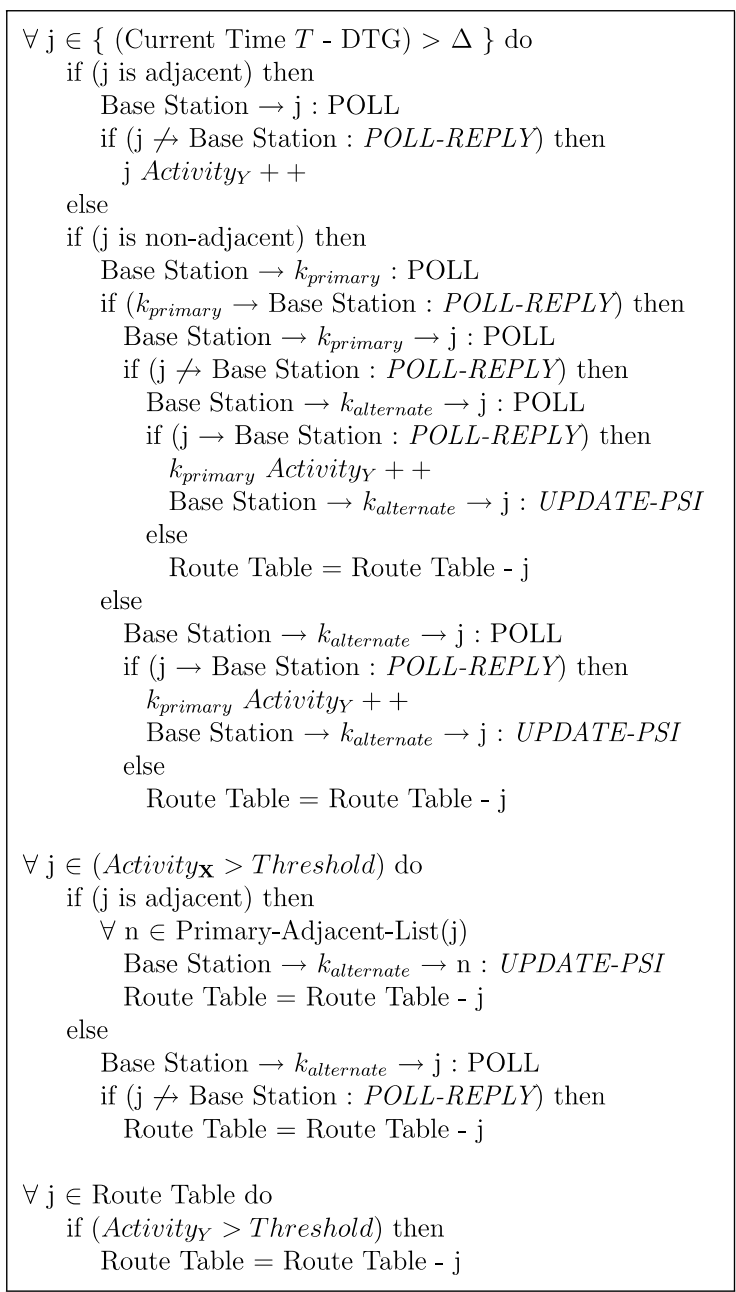

Fig. 7. Network repair protocol. errant behavior on the part of the node. If the node relies upon an intermediate node this could be evidence of aberrant behavior on either the part of the node or the intermediate node. In either case, the base station POLLs the node.

In the case of an adjacent node, if the base station does not receive a $P O L L-R E P L Y$ within a specified time out period $t$, it increments its counter of route failures (Activity ${ }_{Y}$ ) for that node.

In the case of a non-adjacent node, the base station first polls the primary intermediate node. If it receives a $P O L L-R E P L Y$, it polls the node via the primary. Receipt of a $P O L L-R E P L Y$ indicates that everything is in order. The non-receipt of a POLL-REPLY causes the base station to POLL the node via an alternate path, if it exists. A response from the non-adjacent node via the alternate path prompts the base station to transmit an $U P D A T E-P S I$ to it and increment Activity $_{Y}$ for the primary. A non-response causes the base station to delete the non-adjacent node from its route table.

If the base station did not receive a POLL$R E P L Y$ from the primary intermediate node, it $P O L L$ s the non-adjacent node via an alternate path, if it exists. Upon receipt of a POLL-REPLY, it transmits an UPDATE-PSI message, which reflects the alternate route, to the non-adjacent node and increments Activity $_{Y}$ for the primary. If it does not receive a POLL-REPLY from the nonadjacent node via the alternate path, it deletes that node from its route table.

The base station also checks for a high incidence of corrupted messages originating from a non-adjacent node. An intermediate node that has become aberrant may corrupt data that it is relaying on behalf of a non-adjacent node. This behavior constitutes a denial-of-service (DoS) attack. In order to mitigate against such an intermediate node, the base station keeps a counter of corrupted packets, Activity ${ }_{X}$. When this counter crosses a pre-determined threshold, the base station transmits an UPDATE-PSI to all non-adjacent nodes affected by the intermediate node via alternate paths, if they exist, and deletes the aberrant intermediate node from its route table. A non-adjacent node may be sending corrupt data which the intermediate faithfully relays. The base station 
tests for this situation by transmitting a POLL to the non-adjacent node via an alternate path, if it exists. Non-receipt of a POLL-REPLY from the non-adjacent node causes it to be deleted from the base station's route table; receipt indicates that everything is in order.

\subsection{Comparison with SPINS}

SPINS is comprised of Sensor Network Encryption Protocol (SNEP) and Micro Timed Efficient Stream Loss-tolerant Authentication ( $\mu$ TESLA). The function of SNEP is to provide confidentiality (privacy), two-party data authentication, integrity and freshness. $\mu$ TESLA is to provide authentication to data broadcasts. We compare our security model to SNEP in terms of functionality. We do not compare it to $\mu$ TESLA because we do not perform broadcast authentication.

In SNEP each node $j$ shares a unique master key $K_{j}$ with the base station. This master key is used to derive all other keys. For data encryption SNEP employs a one time encryption key produced by using a key derived from $K_{j}$ and an incremental counter (message indicator) as inputs to the RC5 cryptographic algorithm. The RC5 algorithm outputs a binary string that is used as the one time key. The message is XORed with the one time key, transmitted and the counter is incremented in preparation for the next message. The base station, aware of the node's counter value and the derived key, produces the identical one time key, XORs the encrypted message with the one time key to produce the clear text.

Our protocol differs from SPINS in the following fundamental and essential ways:

1. SPINS uses source routing, which like all nonbroadcast routing mechanisms, is vulnerable to traffic analysis. Our protocol relies upon broadcasts where the entire communication is end-to-end encrypted in order to mitigate against the threat posed by traffic analysis.

2. We provide a mechanism for detecting certain types of aberrant behavior, behavior that may be due to either a compromise or malfunction of an individual node. In either case we are able to remove the node from the network. This is extremely important in the case of applications such as perimeter protection, because the longer a compromised node remains in the network, the greater its chances of being able to break down the perimeter.

3. SPINS has been highly optimized in order to reduce code footprint on the SmartDust Mote. Although, our model has not been optimized for a specific sensor platform, the encryption algorithm may be replaced to better suit different hardware platforms.

In addition, we note that our protocol differs from SPINS in terms of the application class. Our protocol is designed to function correctly in sensor networks used for perimeter protection.

\section{Implementation details and simulation results}

We have implemented the topology discovery and network setup components of our protocol using the SensorSim [4] extension of the NS network simulator [18]. The entire protocol, to include cryptographic functionality is implemented at the routing layer. We generated 100 cryptographic keys, where the first key was used as the $\boldsymbol{K} \boldsymbol{e} \boldsymbol{y}_{\mathrm{BS}}$. These keys were placed in the base station. Individual keys and $\boldsymbol{K e y}_{\mathrm{BS}}$ were also placed in each node. Topology distribution files, consisting of the $(x, y)$ co-ordinates of each node were created for four different distributions, which we explain shortly. Each distribution file and a configuration file were used as inputs to the simulator. The correctness of the topology discovery and network setup protocol was verified by observing the successful encryption, transmission, reception, decryption and response to each message. Additionally, the routing table created by the base station was visually inspected to verify that each adjacent node was associated with approximately the same number of non-adjacent nodes. The correctness of the network repair protocol was verified by observing the base station's response to node failures and corrupted data. 


\subsection{Simulation}

We used the simple battery, radio and CPU models of the simulator. According to the battery model, the initial energy of the battery attached to each sensor node is $36 \mathrm{As}(10 \mathrm{~mA} \mathrm{~h})$. According to the radio model, message transmission and reception at a data rate of $19.2 \mathrm{Kbps}$ draw 5.2 and 4.1 $\mathrm{mA}$ of current, respectively. The model assumes a radio range of $15 \mathrm{~m}$. The CPU model assumes that the CPU draws $2.9 \mathrm{~mA}$ in active mode and $1.9 \mathrm{~mA}$ in sleep mode. For purposes of this simulation, we assume a voltage of $1 \mathrm{~V}$. It should be noted that the CPU is assumed to draw a constant amount of current in active mode, irrespective of the task it performs, be it a simple arithmetic function or encryption of a message. We assume a message length of either 24 or 48 bytes.

We conducted experiments to measure energy expenditure of each sensor function ( $T x, R x$ and $C P U$ ) during network setup time for four different network topologies. We simulated a geographic environment measuring $5654 \mathrm{~m}^{2}$. We divided this environment into two concentric circles, the inner circle with a radius of $15 \mathrm{~m}$ and the outer circle with a radius of $30 \mathrm{~m}$. The base station was located at the center. The sensor placement within our experimental topologies is as follows:

1. 30 nodes randomly placed in the inner circle and 70 nodes randomly placed in the outer circle;

2. 50 nodes randomly placed in the inner circle and 50 nodes randomly placed in the outer circle;

3. 70 nodes randomly placed in the inner circle and 30 nodes randomly placed in the outer circle;

4. 100 nodes randomly placed across the entire area.

The results of our experiments are illustrated in the graphs contained in Fig. 8. We measured the energy consumed ( $Y$ axis) by each component of the sensor: the transmitter, receiver and CPU, for the entire network of 1 base station and 99 sensors, plotting it against the time taken ( $X$ axis) for the particular network topology to converge. We used a log plot so that small values would be discernible.

Accordingly, Fig. 8(a) shows the results for Topology \#1. Topology discovery and network setup occurred in $54 \mathrm{~s}$ of simulation time with a total energy expenditure of $37.8 \mathrm{~mJ}$ for all nodes in the sensor network. Fig. 8(b) shows the results for Topology \#2. It took $55 \mathrm{~s}$ of simulation time for topology discovery and network setup and the total network energy consumption was $38.5 \mathrm{~mJ}$. The energy expenditure and time taken for Topology \#3 is illustrated in Fig. 8(c). Here energy consumption was $22.4 \mathrm{~mJ}$ and it took $32 \mathrm{~s}$. The random distribution of sensors in Topology \#4 took $75 \mathrm{~s}$ of simulation time and energy consumption was $52.5 \mathrm{~mJ}$.

In all cases, and as anticipated, the receiver $(R x)$ component consumed the highest amount energy, closely followed by the CPU. The transmitter $(T x)$ component consumed the least amount of energy. This is intuitive, as the number of messages received greatly outweighs those transmitted.

The topology with the densest inner circle and the sparsest outer circle consumed the least amount of energy and converged the quickest. The topology scenario that was most representative of the methods used for physical protection (30 inner nodes and 70 outer nodes) was near the median for time and energy consumption. Our results indicate that as the ratio of adjacent to non-adjacent nodes increases in favor of adjacent nodes, energy consumption for topology discovery and network setup decreases.

The cost of network setup, in terms of energy consumption, is the most expensive period due to the volume of messages. However, energy consumption decreases from this point forward for the life of the sensor network. To put the energy requirements into perspective, suppose that a sensor network using our security protocol were to maintain its peak rate for a protracted period. If this were the case then each sensor equipped with a battery similar to the Eveready $X 91$ with a capacity of $3135 \mathrm{mAh}$ would sustain it for approximately $435 \mathrm{~h}$. (Note: the Berkeley Renee Mote uses two of these batteries [19].) As our network would have a required lifespan of a few days, this 

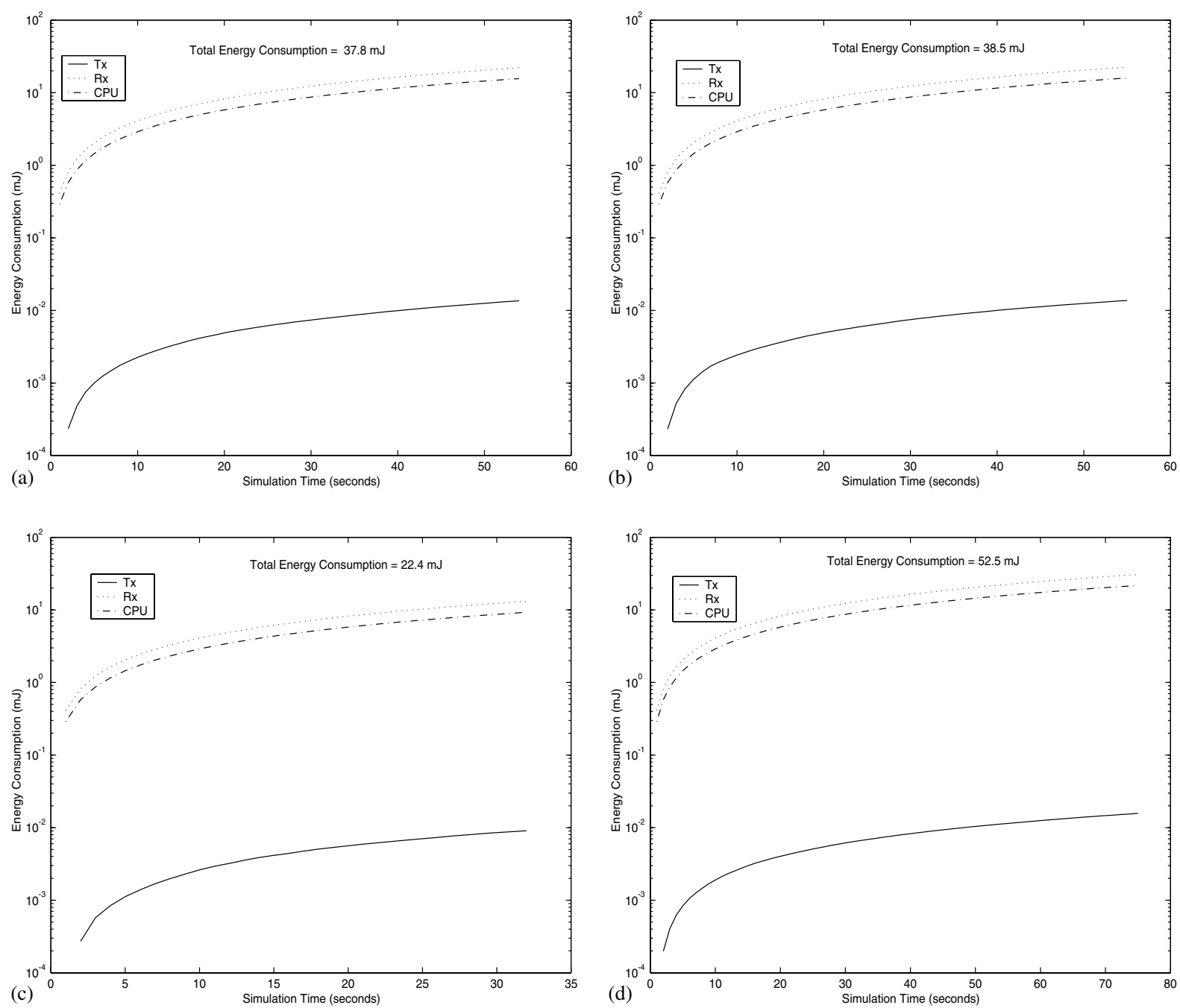

Fig. 8. Simulation results of the topology discovery and network setup protocol: (a) Topology \#1, (b) Topology \#2, (c) Topology \#3, (d) Topology \#4.

time period is well within the bounds of the requirements for our application class.

We have also implemented the protocol to repair the network from the effects of an aberrant node. In this paper, we concentrate on simulating the effects of nodes that have not been active for the last $\Delta$ time units. We chose values of 15,5 and 5 for $\Delta, X$ and $Y$, respectively. The simulation consisted of causing five adjacent and five nonadjacent nodes to become inactive during the simulation, for each of four topologies described above. During the first $3 \mathrm{~s}$ of the simulation, and periodically afterwards, the sensors transmit data to the base station, which populates the Activity Table accordingly. For the remaining simulation time period, the base station periodically launches the network repair protocol described in Fig. 7 to update the routing table and transmit UPDATEPSI messages as required.

The main reason for the short simulation time is the constant (simulation) time of $0.03 \mathrm{~s}$ required by the base station for polling each node and repair- 

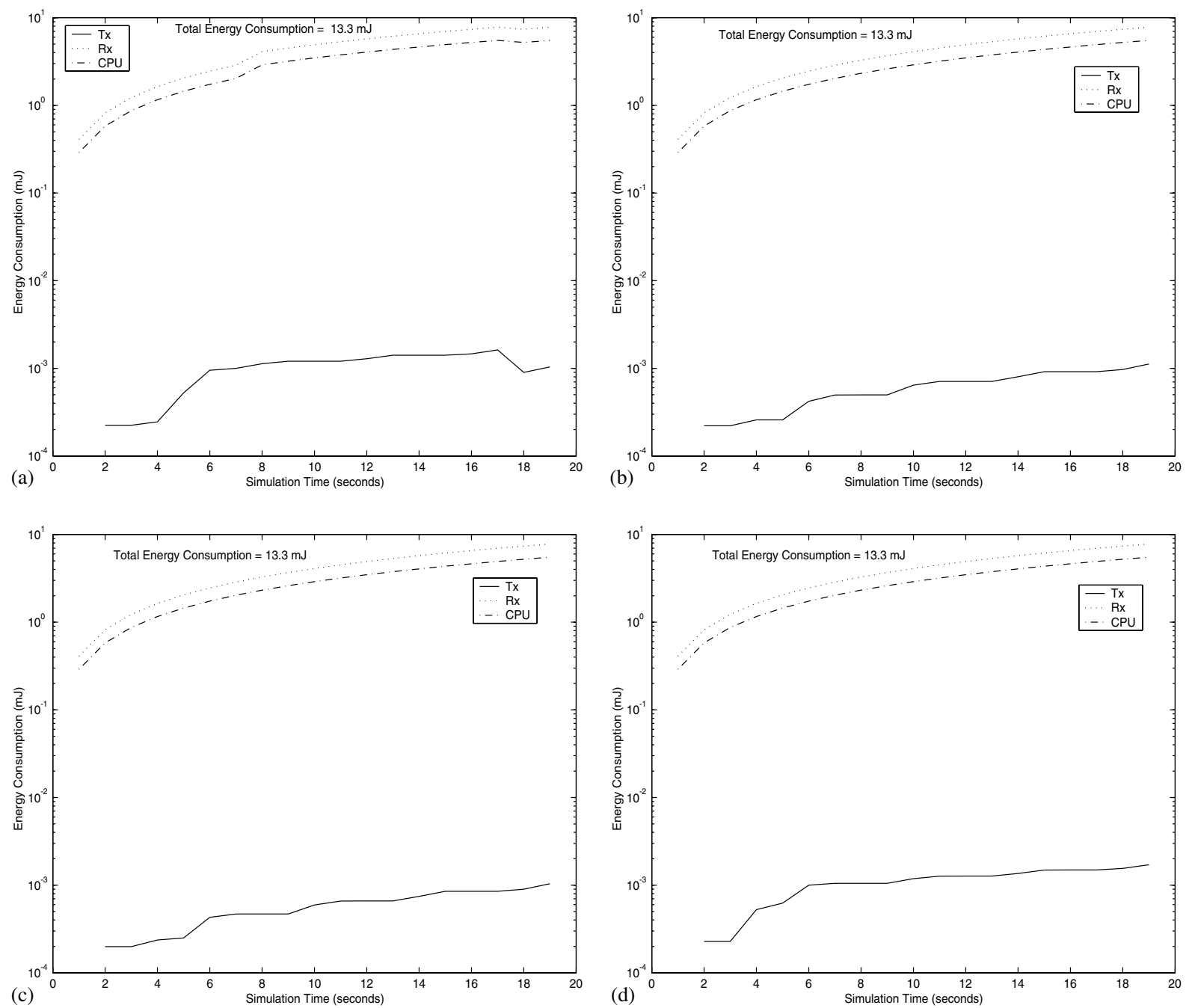

Fig. 9. Simulation results of the network repair protocol: (a) Topology \#1, (b) Topology \#2, (c) Topology \#3, (d) Topology \#4.

ing the network based on the responses (either direct or relayed). For each topology, this time corresponds to energy consumption of approximately 7.7 mJ. Fig. 9(a-d) shows the energy consumption over the $20 \mathrm{~s}$ simulation time period for each of the four topologies.

\section{Conclusions and future work}

A novel scenario defining perimeter protection as an application class of sensor networks was presented. We identify threats to this application class and proposed and implemented a new security model, comprised of the topology discovery and network set protocol and the network repair protocol. We simulated our model using SensorSim and the results indicate that it is viable and well suited for our application class.

As part of our future work, we propose to use other battery models (such as the Lithium and Coin Cell models) and radio propagation models (such as two ray ground and TDMA). Finally, we note that we have designed our security model for 
the specific purpose of perimeter protection. We would like to extend it to take into account requirements of other related applications in order to make it more generalized.

\section{Acknowledgements}

This work was supported in part by NSF awards IIS 9875433 and CCR 0070802 and Fujitsu Laboratories of America, Inc.

\section{References}

[1] Ball Semiconductor Inc., Medical Applications-Benefits of Spherical Geometry, Ball Semiconductor, Inc, 1997. Available from <http://www.ballsemi.com/medical/MedPage.html>.

[2] J.M. Kahn, R.H. Katz, K.S.J. Pister, Mobile networking for smart dust, in: ACM/IEEE Intl. Conf. on Mobile Computing and Networking, 1999.

[3] A. Perrig, R. Szewczyk, V. Wen, D. Culler, J.D. Tygar, SPINS: security protocols for sensor networks, Wireless Networks 8 (2002) 521-534.

[4] S. Park, A. Savvides, M.B. Srivastava, Sensorsim: a simulation framework for sensor networks, in: Proc. of ACM MSWiM 2000, 2000, pp. 104-111.

[5] S. Yi, P. Naldurg, R. Kravets, Security-aware ad hoc routing for wireless networks, in: Proc. of 2001 ACM International Symposium on Mobile Ad Hoc Networking and Computing, 2001, pp. 299-302.

[6] D.E. Bell, L.J. LaPadula, Secure computer systems: mathematical foundations and model, Technical Report M74-244, Mitre Corporation, 1975.

[7] Y. Kostov, G. Rao, Low cost optical instrumentation for biomedical measurement, J. Rev. Sci. Instrum. (2000) 4361-4373.

[8] C. Intanagonwiwat, R. Govindan, D. Estrin, Directed diffusion: a scalable and robust communication paradigm for sensor networks, in: Proc. of Mobicom '00, 2000.

[9] J. Kulik, W.R. Heinzelman, H. Balakrishnan, Adaptive protocols for information dissemination in wireless sensor networks, in: Proc. of Mobicom '99, Seattle, WA, 1999.

[10] S. Madden, M.J. Franklin, Fjording the stream: an architecture for queries over streaming sensor data, in: Proc. of ICDE 2002, 2002.

[11] P. Bonnet, J.E. Gehrke, P. Seshadri, Towards sensor database systems, in: Proc. of Second International Conference on Mobile Data Management, 2001.

[12] National Institute of Standards and Technology, FIPS 140-2; Security Requirements for Cryptographic Modules, November 2002.
[13] National Institute of Standards and Technology, FIPS 462; Data Encryption Standard, December 1993.

[14] National Institute of Standards and Technology, FIPS 81; DES Modes of Operation, December 1980.

[15] D. Denning, Cryptography and Data Security, AddisonWesley, Boston, MA, 1982.

[16] B. Schneier, Applied Cryptography, second ed., Wiley, 1996.

[17] X. Chen, T. Woo, Energy Efficient Data Encryption Algorithms, December 2002. Available from <http:// www.vlsi.uwaterloo.ca/thwoo/ece750report.pdf>.

[18] The Network Simulator, 1996. Available from <http:// www-mash.berkeley.edu/ns $>$.

[19] Mote. Available from <http://kingkong.me.berkeley.edu/ nota/RunningMan/Mote.htm>, page from the Smart Dust program giving an overview of the Berkeley Renee Mote.

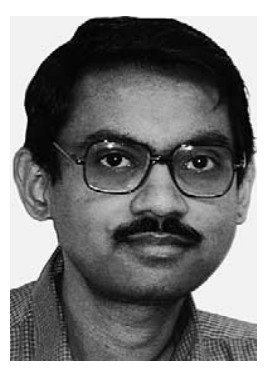

Sasikanth Avancha is a Ph.D. student of Computer Science in the Department of Computer Science and Electrical Engineering at the University of Maryland, Baltimore County. His research interests include wireless sensor networks, security, mobile computing, and distributed systems. He obtained his MS degree in Computer Science from UMBC and his Bachelor of Engineering degree in Computer Science and Engineering from Bangalore University, India. $\mathrm{He}$ has over 5 years of experience in network software development and maintenance.

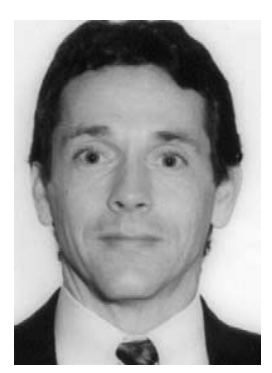

Jeffrey Undercoffer holds a BS in Computer Science and a MS in Software Engineering from the University of Maryland, University College and College Park respectively. Currently he is pursuing a Ph.D. at the Department of Computer Science and Electrical Engineering at the University of Maryland, Baltimore County, where his research is focusing on networking and security. He is a student member of the ACM and IEEE.

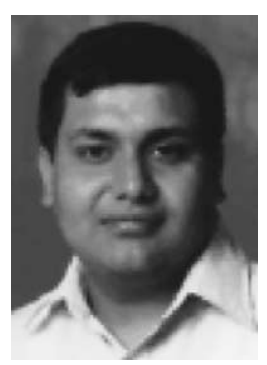

Anupam Joshi is an Associate Professor of Computer Science and Electrical Engineering at UMBC. Earlier, he was an Assistant Professor in the CECS Department at the University of Missouri, Columbia. $\mathrm{He}$ obtained a B.Tech. degree in Electrical Engineering from IIT Delhi in 1989, and a Masters and Ph.D. in Computer Science from Purdue University in 1991 and 1993 respectively. His research interests are in the broad area of networked computing and intelligent systems.

His primary focus has been on data management for mobile 
systems in general, and most recently on data management in mobile ad-hoc networks. He has created intelligent agent based middleware to support mobile access to networked computing and multimedia information resources. He is also interested in Data/Web Mining and Semantic Web, where he has worked on applying soft computing techniques to personalize the Web space. His other interests include content-based retrieval of video data from networked repositories, and networked HPCC. He has published over 50 technical papers, and has obtained research support from NSF, NASA, DARPA, DoD, IBM, AetherSystens, HP, AT\&T and Intel. He has presented tutorials in conferences, served as guest editor for special issues for IEEE Personal Comm., Comm. ACM etc., and serves as an Associate Editor of IEEE Transactions of Fuzzy Systems. At UMBC, he teaches courses in Operating Systems, Mobile Computing, and Web Mining. He is a member of IEEE, IEEE-CS, and ACM.

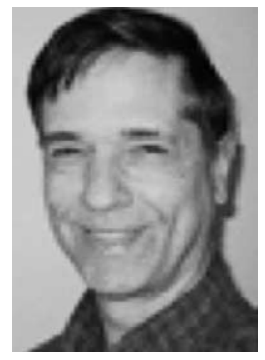

John Pinkston is presently Professor and Chair of the Computer Science and Electrical Engineering Department at UMBC. He received the BSE degree with highest honors from Princeton University in 1964, and the MS and Ph.D. degrees from MIT in 1967, both in Electrical Engineering. He assumed his present appointment at UMBC in September of 1997, following a career in government and industrial research.

His contributions span areas of mathematical cryptology, speech and signal processing, high performance special purpose computers, superdirective antenna arrays, and cryogenic high speed/low power microelectronic devices.

His present research interests include Information Security, Signal Processing, and Communication and Coding Theory. 